\title{
Formation of Periodically-Ordered Calcium Phosphate Nanostructures by Block Copolymer-Directed Self-Assembly
}

Rui-Qi Song, ${ }^{1}$ Tobias N. Hoheisel, ${ }^{1}$ Hiroaki Sai, ${ }^{1}$ Zihui Li, ${ }^{1}$ Joseph D. Carloni, ${ }^{1}$ Suntao Wang, ${ }^{2}$ Randall E. Youngman, ${ }^{3}$ Shefford P. Baker, ${ }^{1}$ Sol M. Gruner, ${ }^{2,4}$ Ulrich Wiesner, ${ }^{1, *}$ Lara A.

$$
\text { Estroff }^{1,4, *}
$$

${ }^{1}$ Department of Materials Science and Engineering, Cornell University, Ithaca, NY, 14853, USA.

${ }^{2}$ Department of Physics, Cornell Unviersity, Ithaca, NY 14853, USA.

${ }^{3}$ Science \& Technology Division, Corning Incorporated, SP-AR-02-4, Corning, NY 14830, USA

${ }^{4}$ Kavli Institute at Cornell for Nanoscale Science, Ithaca, NY 14853, USA

*Correspondence should be addressed to ubw1@cornell.edu, lae37@cornell.edu

\section{Supporting information:}

\section{Figures and Tables}

Fig. S1. SAXS characterization of films obtained from neat polymers IA-1 and IA-2.

Fig. S2. Wide angle $x$-ray diffraction pattern of the osm-ACP nanoparticles.

Fig. S3. Fourier transform infrared spectrum of the osm-ACP nanoparticles.

Fig. S4. ${ }^{31} \mathrm{P}$ MAS NMR spectrum of the osm-ACP nanoparticles.

Fig. S5. ${ }^{29}$ Si CPMAS NMR spectrum of the osm-ACP nanoparticles.

Fig. S6. AFM results for ACP sols obtained without (left) and with (right) the addition of GLYMO.

Fig. S7. TEM characterization of control composites obtained using sols without GLYMO as particle surface modifier.

Fig. S8. Results of DLS measurements.

Fig. S9. 2D SAXS patterns of ACPIA hybrids.

Fig. S10. TEM image of composite ACPIA-2/1 showing large grains.

Fig. S11. SAXS characterization of ACPIA-2/1.

Fig. S12. Representative TEM image of composite ACP-HP-1.

Fig. S13. Representative TEM image of composite ACP-HP-2.

Table S1. Solvent composition of polymer solutions for DLS measurements.

\section{Additional Experimental Details}

a. PDMAEMA homopolymer synthetic details

b. Indexing analysis of the 2D SAXS pattern for ACP/IA2-1

c. Characterization of nanocomposite samples by nanoindentation

d. Calculation of the volume fraction of nanoparticles in the hybrids 


\section{Figures and Tables}
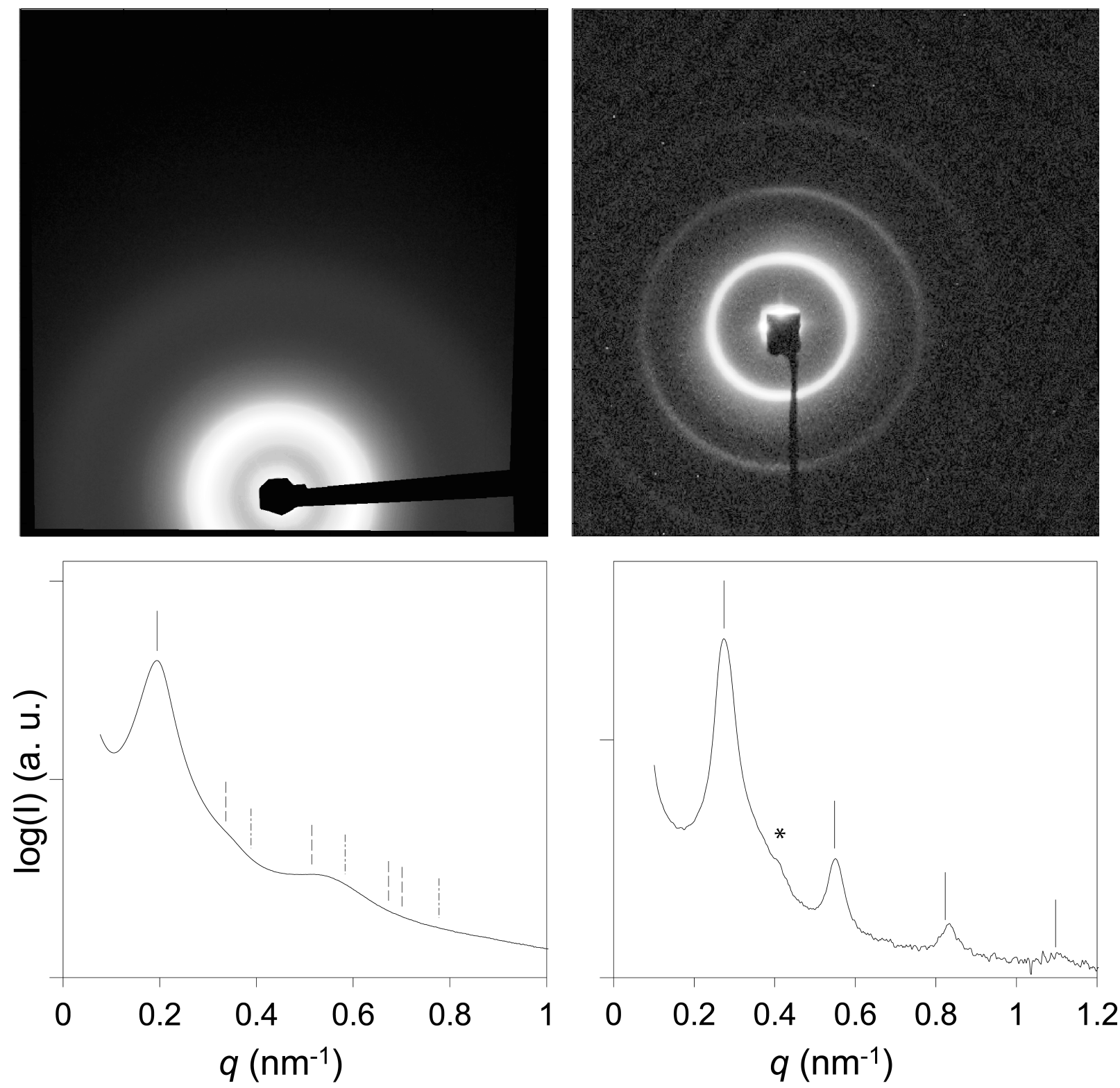

Fig. S1. Characterization of films cast from IA-1 and IA-2 by dissolving $0.03 \mathrm{~g}$ polymer in $4 \mathrm{~mL}$ THF. (Top left) 2-D SAXS pattern of IA-1. (Top right) 2-D SAXS pattern of IA-2. (Bottom left) SAXS profile of IA-1. The solid vertical line corresponds to a characterstic length scale of $32.3 \mathrm{~nm}$. Dashed vertical lines correspond to expected higher order peak positions for a hexagonal lattice. (Bottom right) SAXS profile of IA-2. Vertical lines correspond to expected peak positions for a lamellar lattice with repeat distance of $23 \mathrm{~nm}$. The star indicates a shoulder due to a minor fraction of another lattice. This shoulder may arise from phase modification at the sample surface, which is very common (i.e., see Fig. S9d). 


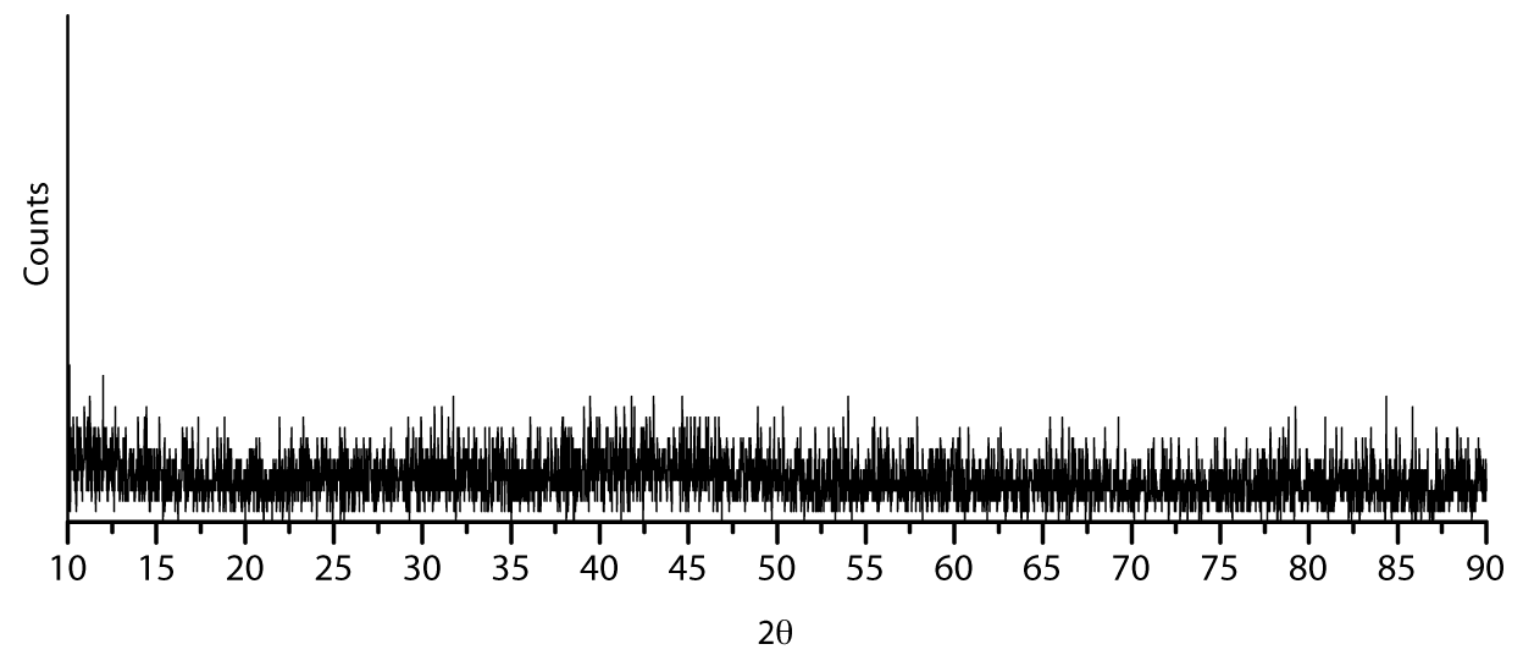

Fig. S2. Wide angle x-ray diffraction pattern (Scintag Theta-Theta X-Ray Diffractometer, $\mathrm{Cu} \mathrm{K} \alpha$, $1.54 \AA$ ) of the osm-ACP nanoparticles demonstrating their amorphous character. 


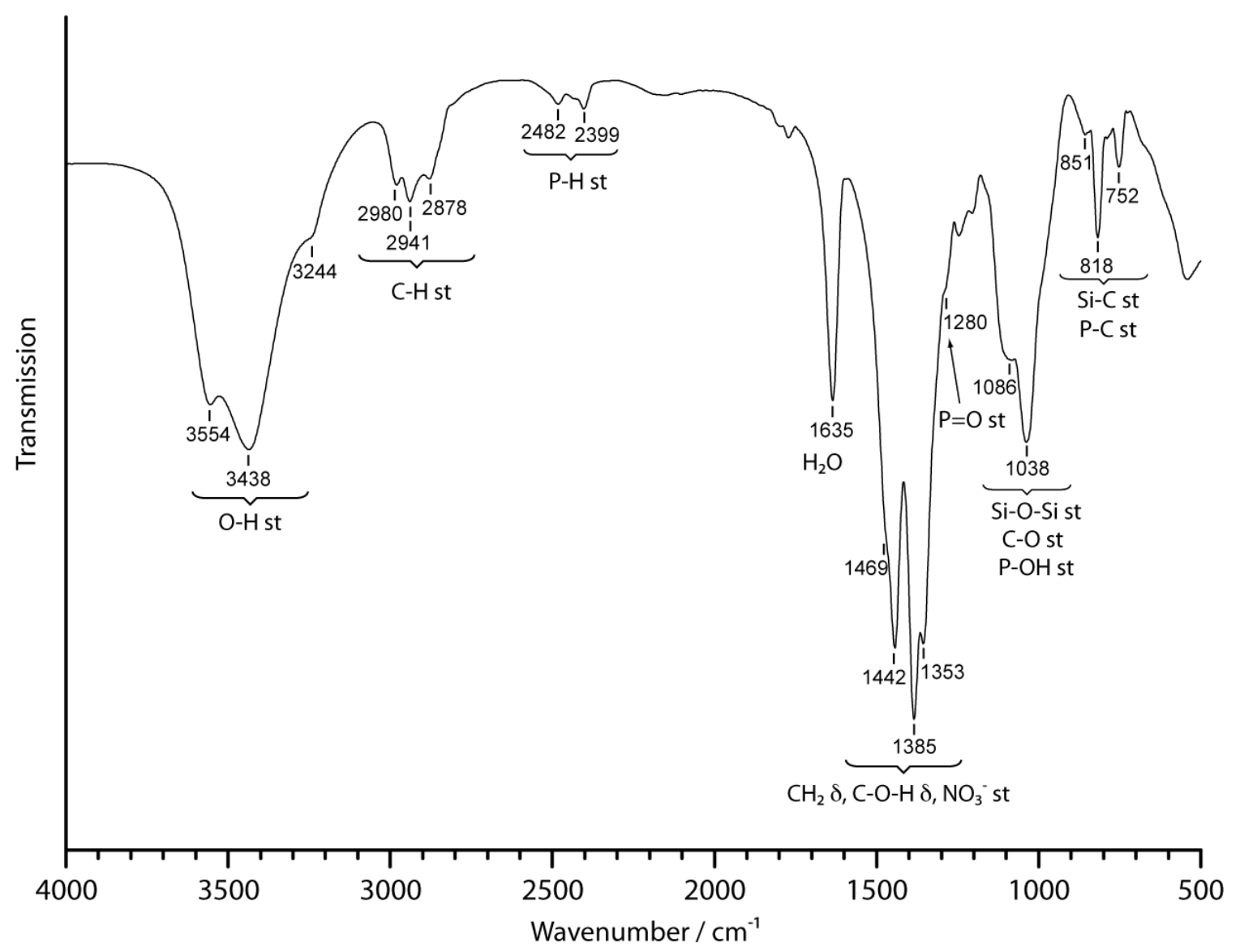

Fig. S3. Fourier transform infrared spectrum (Bruker Vertex 80v equipped with an evacuated sample chamber, recorded at a resolution of $4 \mathrm{~cm}^{-1}$ ) of the osm-ACP nanoparticles dispersed in $\mathrm{KBr}$ (Sigma-Aldrich, FTIR grade) pellet showing the presence of organic moieties, P-O, Si-O, and nitrate. 


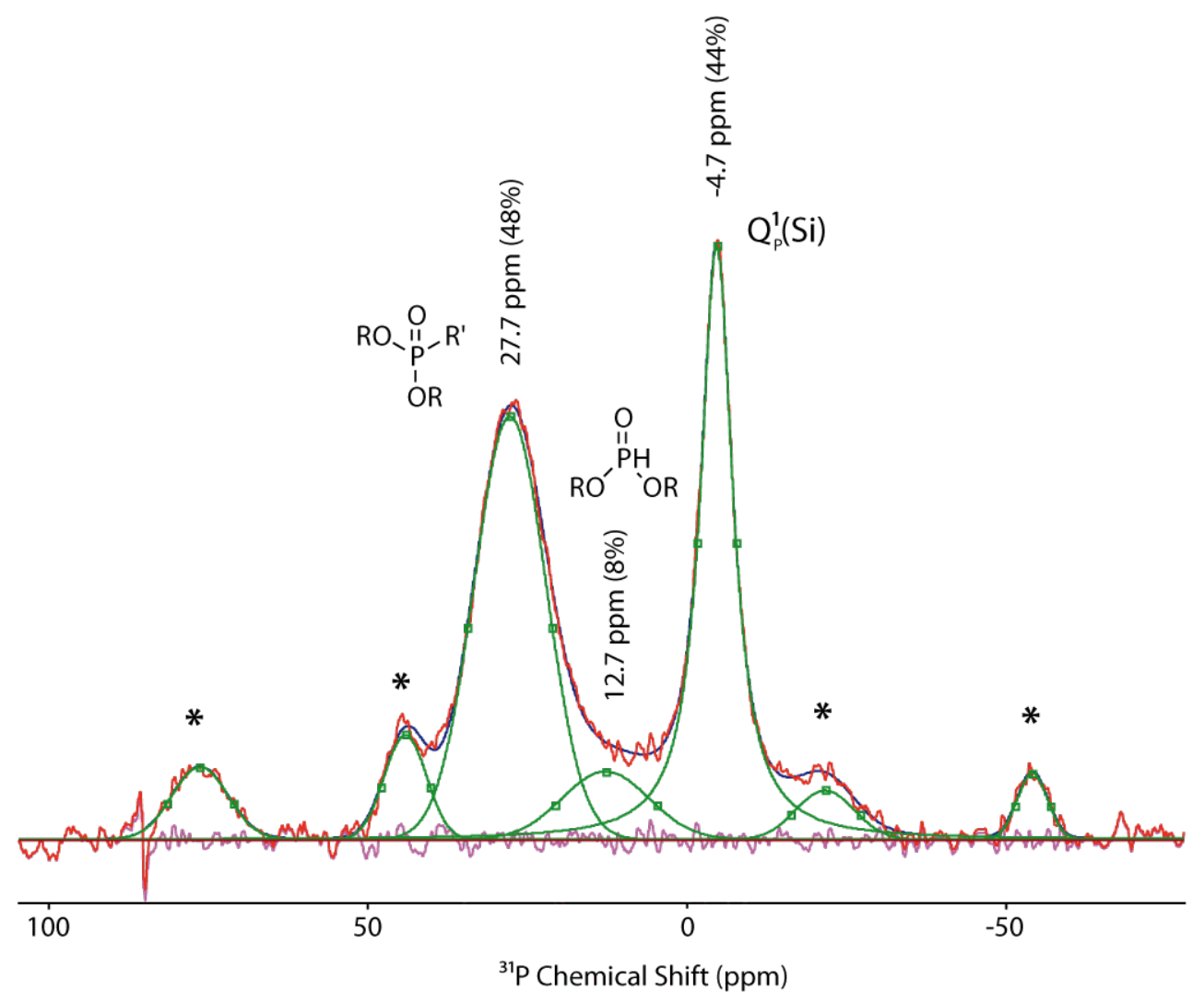

Fig. S4. Solid-state ${ }^{31} \mathrm{P}$ MAS NMR spectrum of the osm-ACP nanoparticles showing the presence of organophosphate groups (most likely phosphonate) at $27.7 \mathrm{ppm}$, orthophosphate groups likely charge-balanced by $\mathrm{Ca}^{2+}$ at $-4.7 \mathrm{ppm},{ }^{1-3}$ and a minor contribution of phosphites at $12.7 \mathrm{ppm}$. The experimental data (red line), was fitted using GRAMS (Thermo Fisher Scientific) using 100\% Gaussian lineshapes (green lines) to fit all resonances except for the peak at -4.7 ppm, which is $100 \%$ Lorentzian. The blue line is the cumulative peak fit, and the pink line represents the residuals. Peaks marked with an asterisk are spinning side bands. MAS spectra were recorded at a resonance frequency of $88.9 \mathrm{MHz}$ and a spinning speed of $4 \mathrm{kHz}$. A single pulse experiment with a pulse width of $2.4 \mu$ s (corresponding to a tip angle of $\pi / 6$ ) with ${ }^{1} \mathrm{H}$ decoupling, $60 \mathrm{~s}$ recycle delay and 1000 acquisitions was performed. The spectrum was processed with $50 \mathrm{~Hz}$ line broadening and chemical shifts are reported relative to $85 \%$ phosphoric acid. 


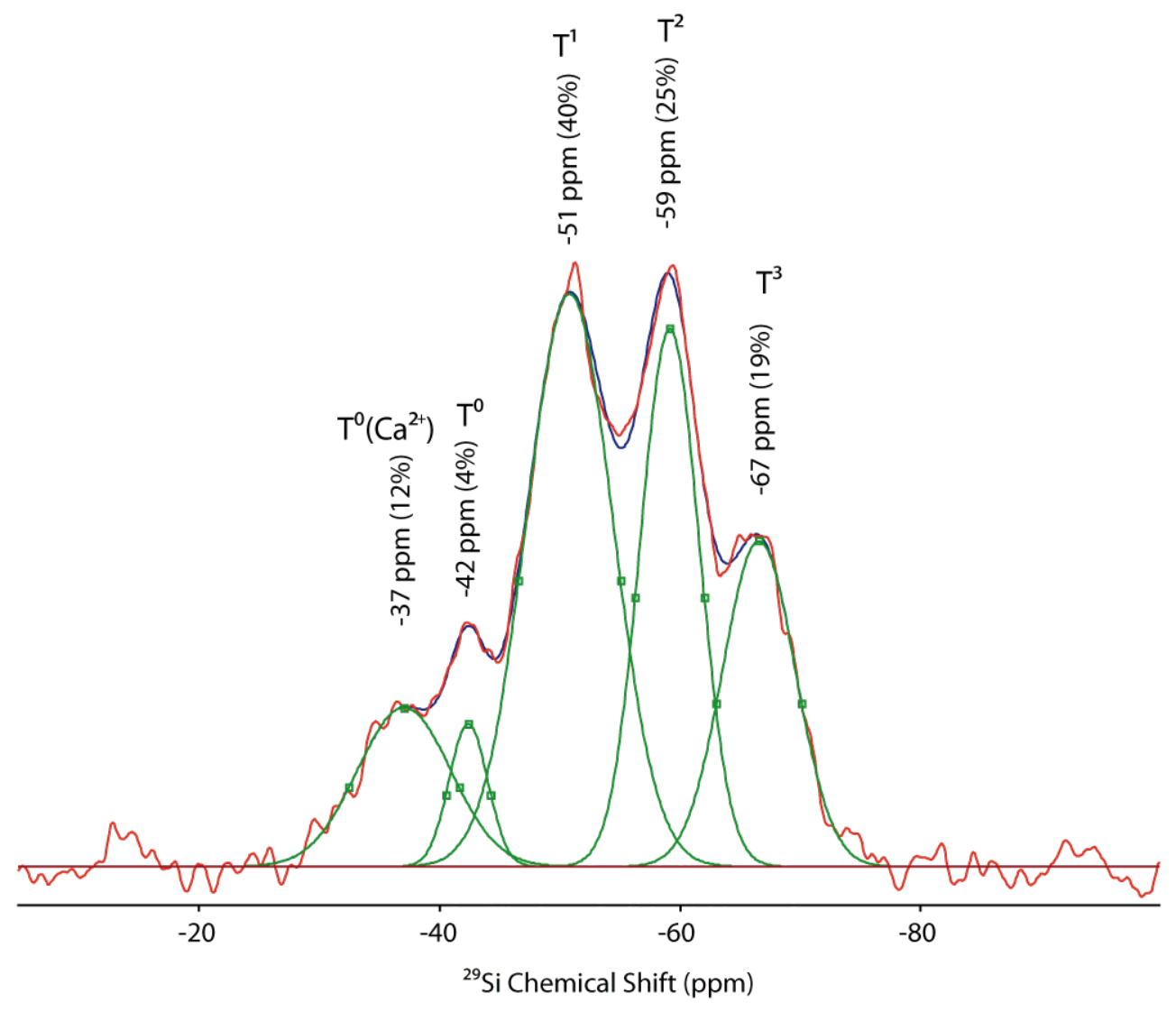

Fig. S5. Solid-state ${ }^{29} \mathrm{Si}$ CPMAS NMR spectrum of the osm-ACP nanoparticles showing the presence of an organosilicate network mainly consisting of $\mathrm{T}^{1}$ and $\mathrm{T}^{2}$ sites, as well as $\mathrm{T}^{3}$ and two different $\mathrm{T}^{0}$ sites, one of which probably is surrounded by $\mathrm{Ca}^{2+}$ inducing a downfield shift. ${ }^{4-7}$ The experimental data (red line), was fitted using GRAMS (Thermo Fisher Scientific) using 100\% Gaussian lineshapes (green lines) and the blue line is the cumulative peak fit. CPMAS spectra were recorded at a resonance frequencies of $99.3 \mathrm{MHz}$ for ${ }^{29} \mathrm{Si}$ nuclei and $499.8 \mathrm{MHz}$ for ${ }^{1} \mathrm{H}$ nuclei, and a spinning speed of $6 \mathrm{kHz}$. The ${ }^{1} \mathrm{H}$ pulse width was $3.8 \mu$ s (corresponding to a tip angle of $\pi / 2$ ) with $1 \mathrm{~ms}$ contact time between ${ }^{1} \mathrm{H}$ and ${ }^{29} \mathrm{Si}$ nuclei, and $15 \mathrm{~s}$ recycle delay. The spectrum was processed with $99 \mathrm{~Hz}$ line broadening and the shifts are reported relative to tetramethylsilane. 

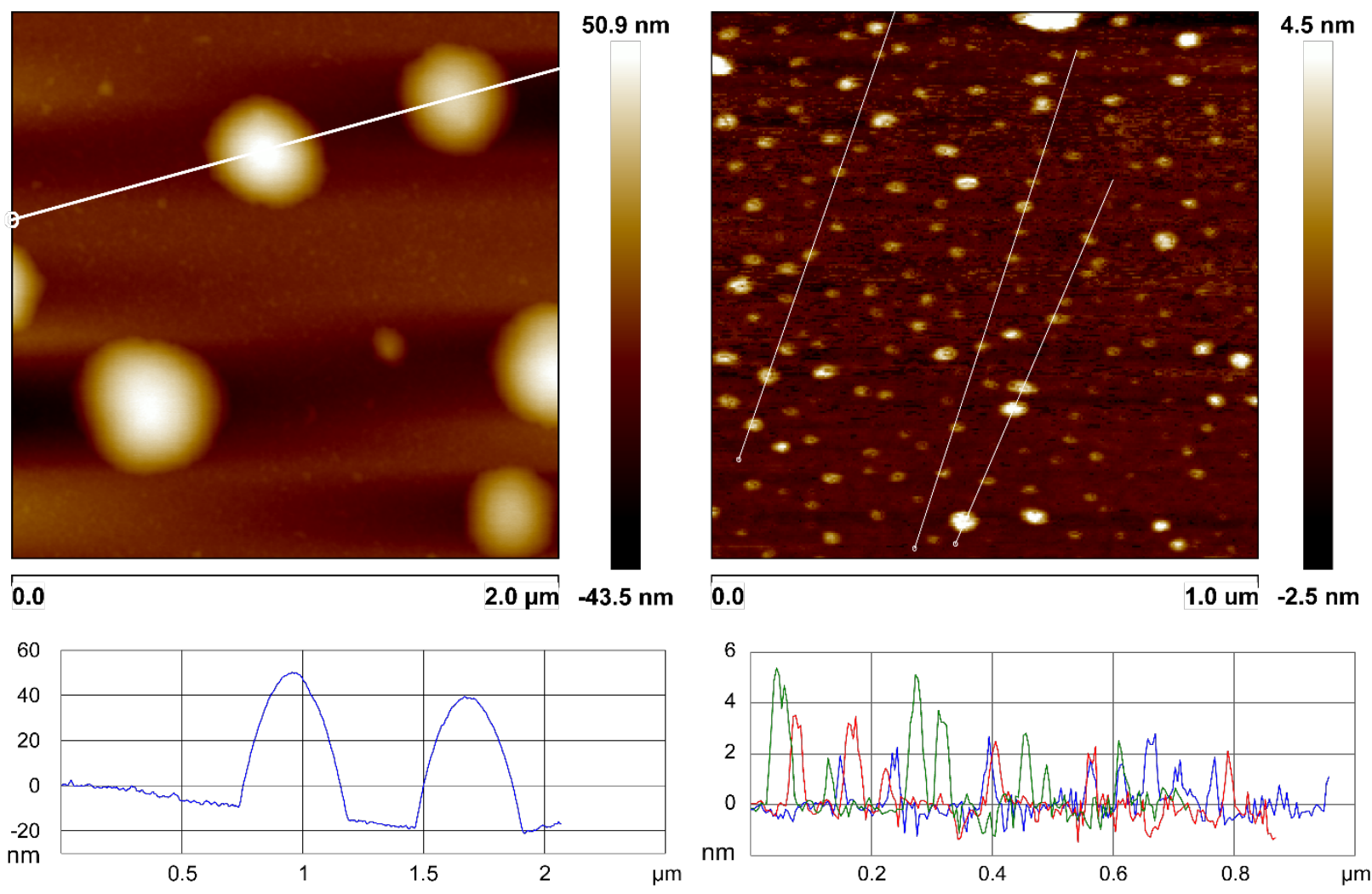

Fig. S6. AFM results for ACP sols obtained without (left) and with (right) the addition of GLYMO. Height profiles for the sections shown above are given in the plots below. 

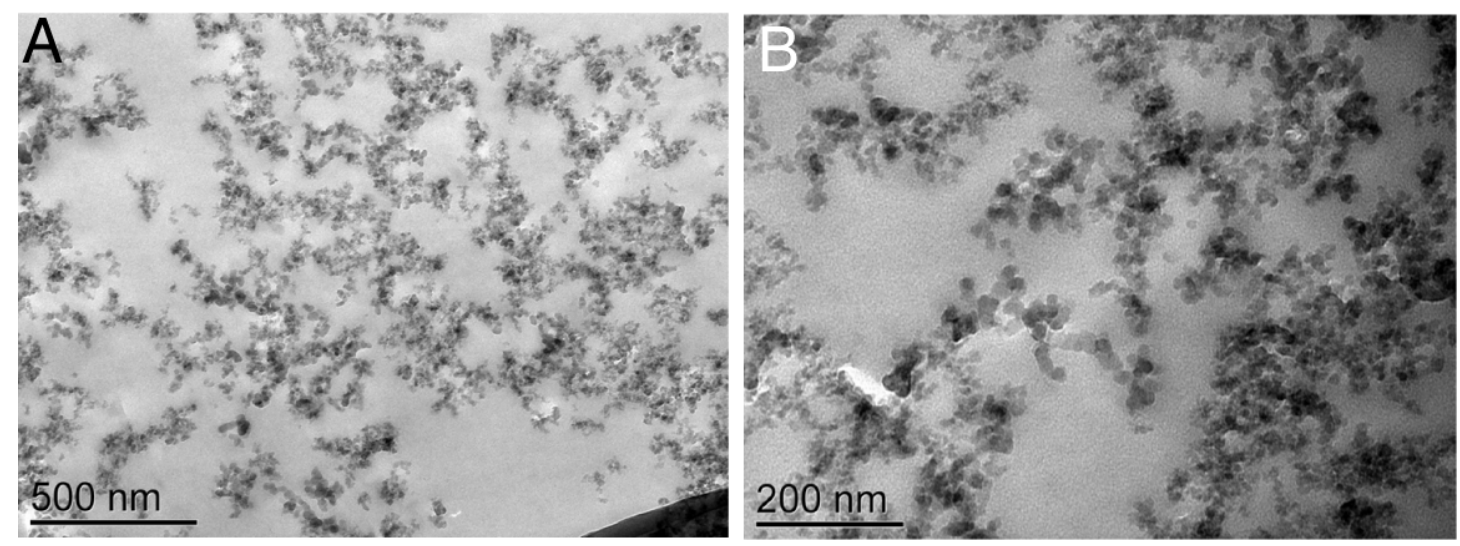

Fig. S7. TEM characterization of unstained control composites obtained using IA-1 and sols without GLYMO as particle surface modifier. 

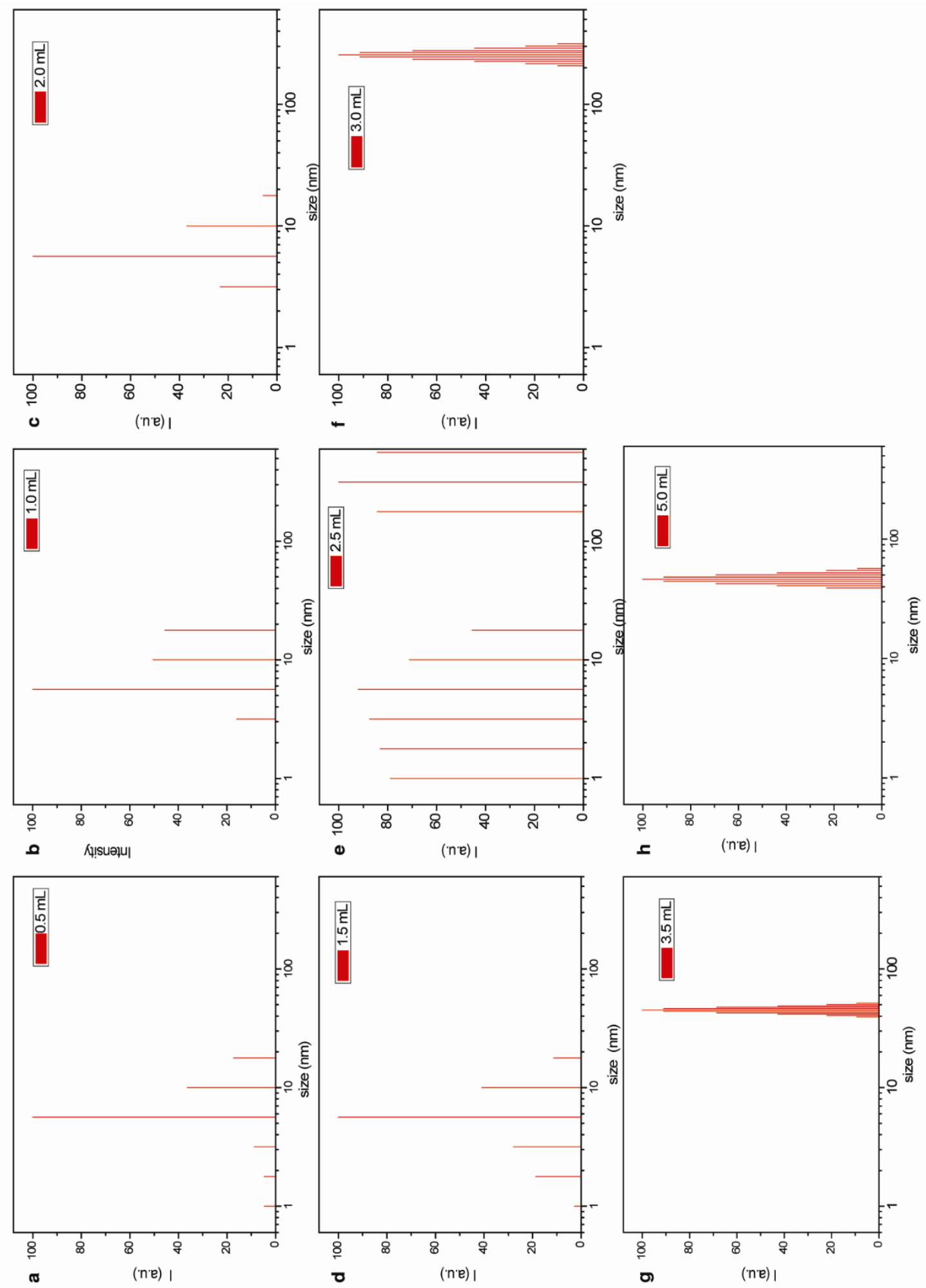

Fig. S8. Results of DLS measurements on solutions of $0.03 \mathrm{~g}$ of IA-1 in $4 \mathrm{ml}$ THF as a function of added co-solvent (1:80 v/v water to ethanol). Amount of added co-solvent is provided in each plot. More details regarding composition of samples are provided in Table S1. 

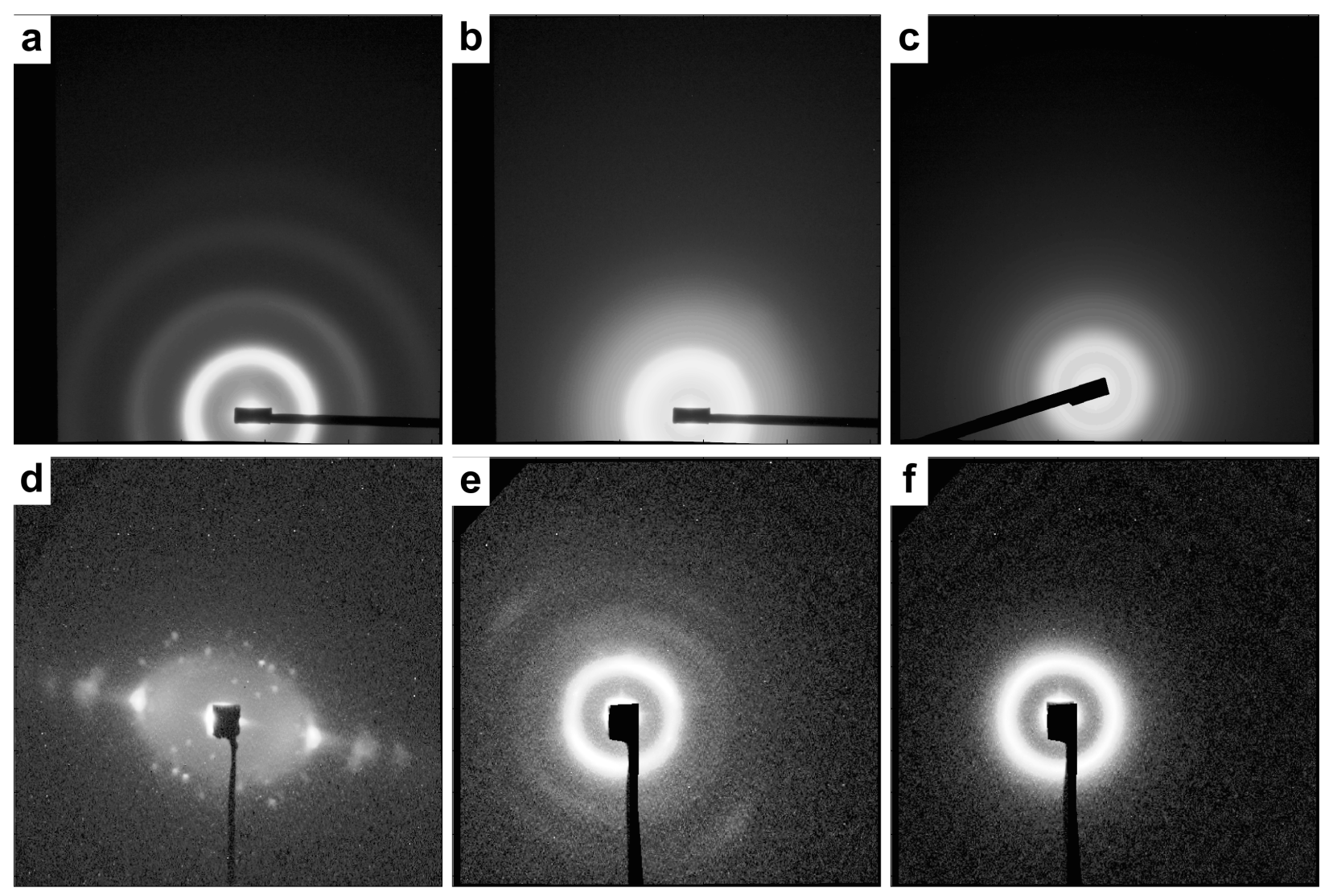

Fig. S9. 2D SAXS patterns of hybrids. (a) ACPIA-1/1, (b) ACPIA-1/2, (c) ACPIA-1/3, (d) ACPIA-2 $/ 1$ with $60^{\circ}$ incident angle for the beam against film normal direction, (e) ACPIA-2/2, and (f) ACPIA-2/3. 


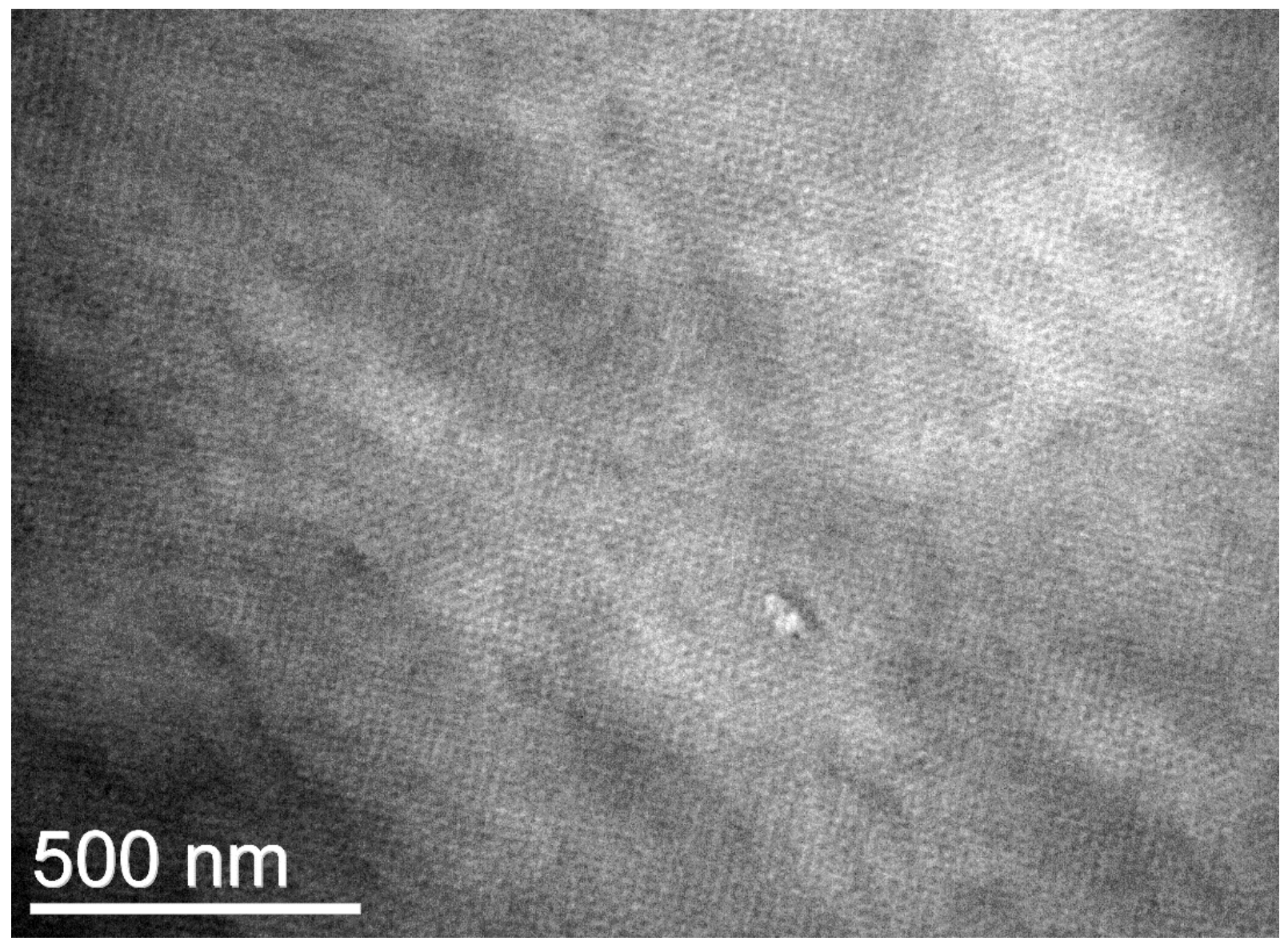

Fig. S10. TEM image of composite ACPIA-2/1 stained with osmium tetraoxide showing large grains. 


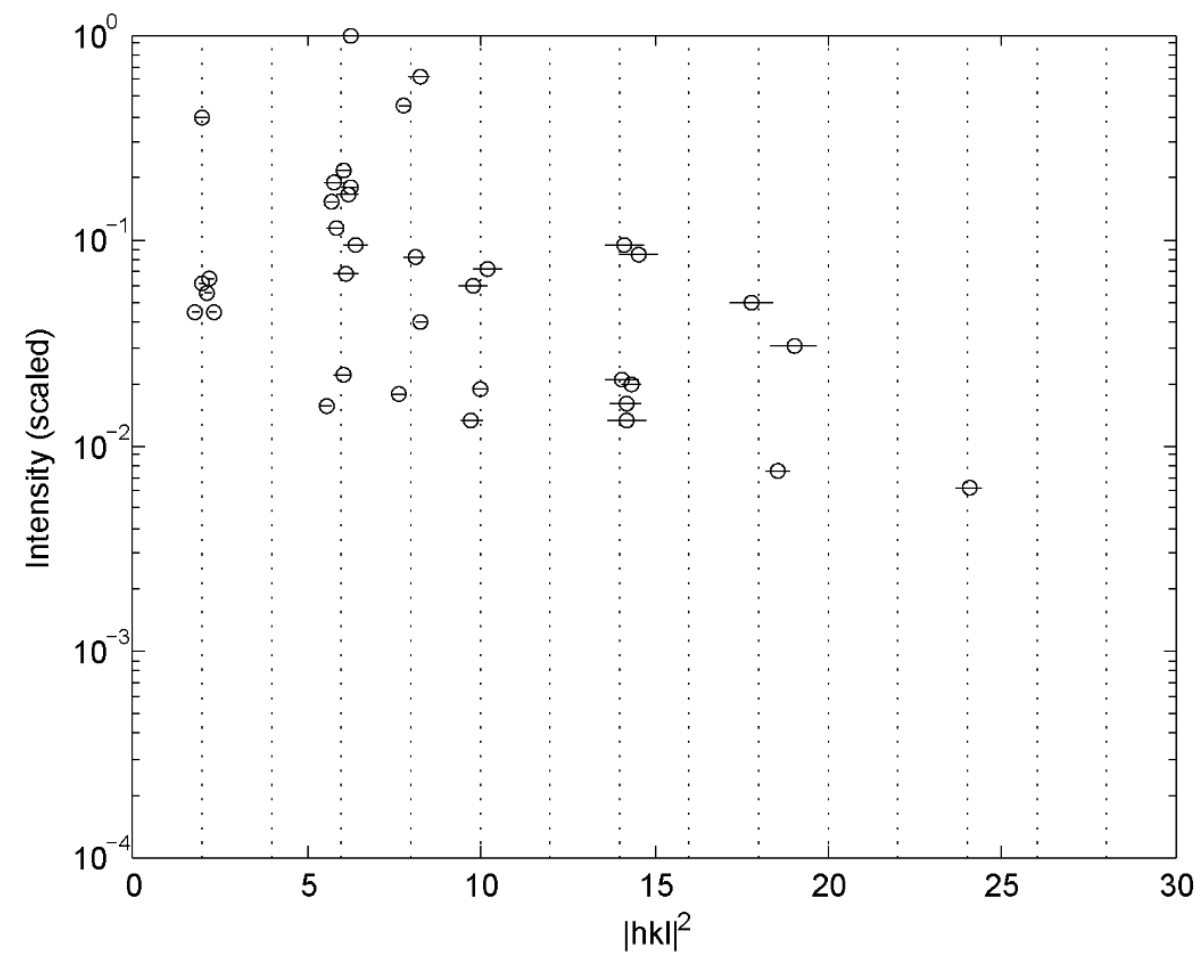

Fig. S11. Spot intensity vs. normalized reciprocal vector magnitude plot for the SAXS pattern shown in Fig. 4g,h. Spots closest to the beam center are indexed as $|h k l|^{2}=h^{2}+k^{2}+l^{2}=2$. The vertical dotted lines show positions for $|h k l|^{2}=2 n$ where $n$ is an integer, which corresponds to reflections from a body-centered cubic family of lattices. 


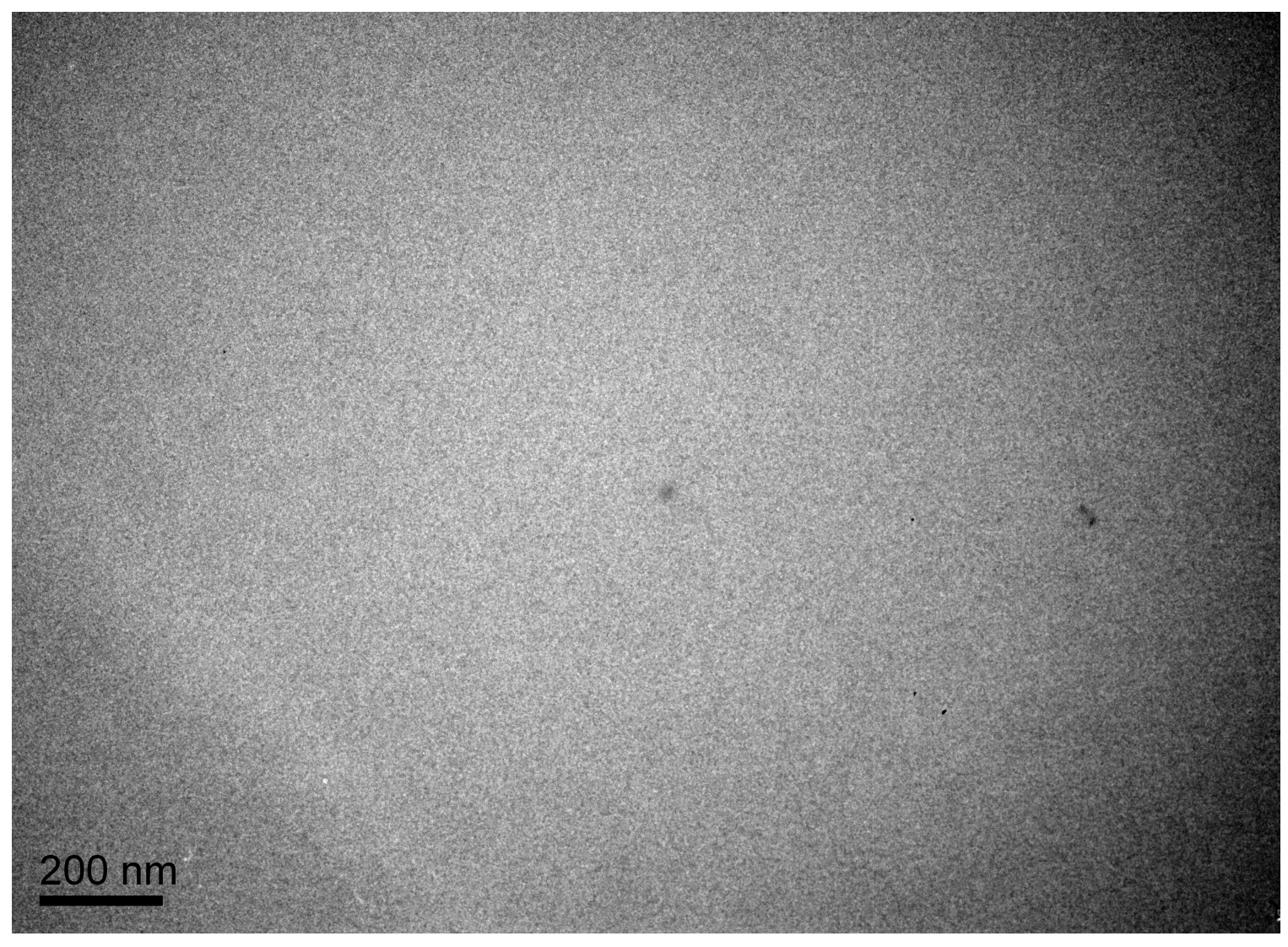

Fig. S12. Representative TEM image of unstained composite ACP-HP-1. 


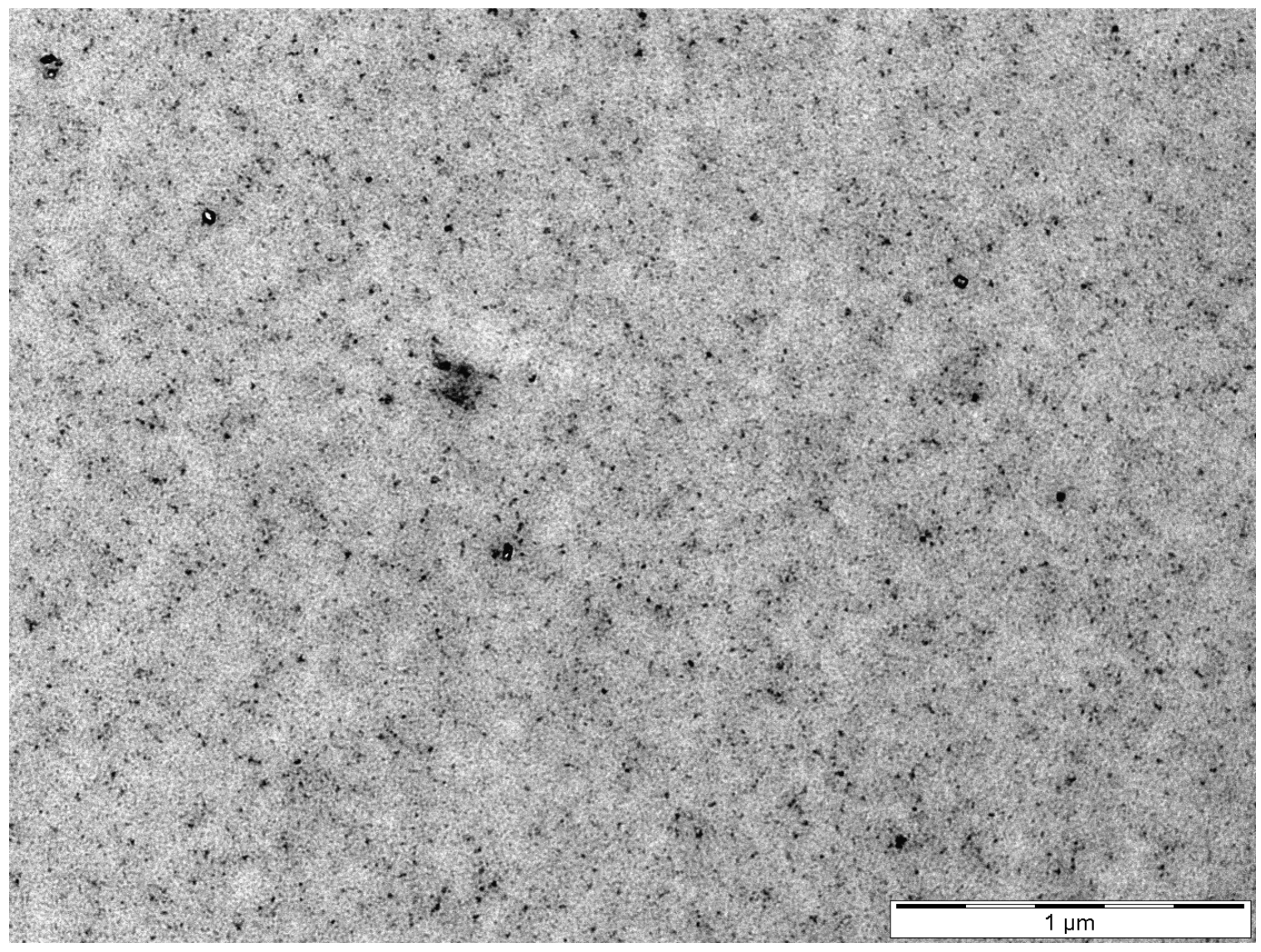

Fig. S13. Representative TEM image of unstained composite ACP-HP-2. 
Table S1. Solvent composition of polymer solutions for DLS measurements.

\begin{tabular}{|c|c|c|c|c|c|}
\hline $\begin{array}{l}\text { Solution } \\
\text { samples }\end{array}$ & $\mathrm{THF} / \mathrm{mL}$ & $\mathrm{H}_{2} \mathrm{O}$-containing ethanol / $\mathrm{mL}$ & $\begin{array}{l}\mathrm{V} \% \\
\left(\mathrm{H}_{2} \mathrm{O}\right)\end{array}$ & $\begin{array}{l}\text { V\% } \\
\text { (Ethanol) }\end{array}$ & $\begin{array}{l}\text { Polymer } \\
\text { structure }\end{array}$ \\
\hline 1 & 4 & 0.5 & 0.14 & 10.97 & Unimers \\
\hline 2 & 4 & 1 & 0.25 & 19.75 & Unimers \\
\hline 3 & 4 & 1.5 & 0.34 & 26.93 & Unimers \\
\hline 4 & 4 & 2 & 0.41 & 32.92 & Unimers \\
\hline 5 & 4 & 2.5 & 0.48 & 37.98 & Aggregates \\
\hline 6 & 4 & 3 & 0.53 & 42.33 & Aggregates \\
\hline 7 & 4 & 3.5 & 0.58 & 46.09 & Micelles \\
\hline 8 & 4 & 5 & 0.69 & 54.87 & Micelles \\
\hline
\end{tabular}

*For each sample, $0.03 \mathrm{~g}$ of IA- 1 was dissolved in $4 \mathrm{~mL}$ of THF. For the water-containing ethanol solution, the volume ratio of $\mathrm{H}_{2} \mathrm{O}$ to THF was 1:80. 


\section{Additional Experimental Details}

\section{a. PDMAEMA homopolymer synthetic details}

PDMAEMA homopolymer was synthesized via anionic polymerization of 2(dimethylamino)ethyl methacrylate (DMAEMA). ${ }^{8}$ DMAEMA (Sigma-Aldrich) was stirred over calcium hydride overnight and degassed in three freeze-pump-thaw cycles followed by distillation. The distillate was filtered in a glovebox over neutral alumina and subsequently stirred over trioctylaluminum (Sigma-Aldrich, $25 \%$ in hexanes) for $5 \mathrm{~min}$, degassed in three freeze-pump-thaw cycles followed by a second distillation. Tetrahydrofuran (THF, J.T. Baker) was distilled over potassium and stirred over $n$-butyl lithium/diphenyl ethylene (DPE, Alfa Aesar) before distillation into a polymerization reactor. The amount of THF was chosen such that a solution with a concentration of less than $10 \mathrm{w} / \mathrm{v} \%$ resulted. For additional purification of the solvent, $1 \mathrm{~mL}$ sec-butyl lithium (Sigma-Aldrich) per $100 \mathrm{~mL}$ THF was added to the THF in the polymerization reactor at $-78{ }^{\circ} \mathrm{C}$, and the mixture was allowed to warm to room temperature under stirring (with a $3 / 8$ " x $7 / 4$ " stir bar) until the yellow color vanished. The mixture was then cooled to $-78^{\circ} \mathrm{C}$ again and stirred vigorously without splashing. An excess of DPE was added followed by the required amount of sec-butyl lithium as initiator, which resulted in an intense red color. The required amount of distilled DMAEMA was then added at $-78^{\circ} \mathrm{C}$ in a constant stream of drops resulting in an immediate decolorization of the solution. The cooling bath was exchanged to an isopropanol/ethylene glycol $(1: 1)$ bath at $-50{ }^{\circ} \mathrm{C}$, and the mixture was stirred for $2.25 \mathrm{~h}$ at this temperature before it was quenched with degassed methanol. The solvent was evaporated, the residue was dissolved in chloroform and the resulting solution was washed three times with $1 \mathrm{M} \mathrm{NaOH}$ (in 1:4 deionized water/saturated sodium chloride solution) and once with saturated sodium chloride solution. The organic phase was evaporated yielding PDMAEMA as a colorless foam. Gel permeation chromatography (GPC) was used to determine the polydispersity index (PDI) of the polymer and ${ }^{1} \mathrm{H}$ NMR spectroscopy was used to determine its overall molar mass. The PDMAEMA homopolymer had a molar mass of $53.8 \mathrm{~kg} / \mathrm{mol}$ and a PDI of 1.04 with a small low molar mass impurity at $7 \mathrm{~kg} / \mathrm{mol}$ and may have contained up to $5 \mathrm{wt} \%$ of inorganic component, most likely sodium chloride. 


\section{b. Indexing analysis of the 2D SAXS pattern for ACP/IA2-1 (Fig. 4h)}

Indexing of Bragg spots in the 2D SAXS pattern for ACP/IA2-1 was performed via three steps as described by Finnefrock and Toombes ${ }^{9-11}$ :

1. Peak position and intensity identification,

2. Beam center and lattice dimension refinement using ellipticity compensation,

3. Iterative identification of zone axes that yield the largest subset of observed peaks.

36 peaks were identified for this pattern, and an automatic matching algorithm scanned the zone axis direction to identify the red [11ㅣ], green [111], and blue [528] grains (colors refer to Fig. 4h) that span the entire set of reflections observed, with a mosaic spread of $5^{\circ}$. Note that this set of grain orientation assignment is not unique: however it does not affect our conclusion that a rather small number of grains exist within the interaction volume.

\section{c. Characterization of nanocomposite samples by nanoindentation:}

Samples (100's of $\mu \mathrm{m}$ thick and several $\mathrm{mm}^{2}$ ) were embedded in epoxy resin (EpoxyMount two component epoxy resin from Allied High Tech Products Inc., Rancho Dominguez, CA). After hardening, the block copolymer-nanoparticle samples were polished

using $3 \mu \mathrm{m}$ and $1 \mu \mathrm{m}$ diamond lapping films (Allied High Tech Products inc., Rancho Dominguez, CA) at 50 RPM and subsequently using a $0.1 \mu \mathrm{m}$ diamond lapping film (Allied High Tech Products Inc., Rancho Dominguez, CA) at 30 RPM on a Multiprep Polisher (Allied High Tech Products Inc., Rancho Dominguez, CA) to expose the surface. The surfaces of all BCP hybrid samples in epoxy were further treated to obtain a RMS roughness of $<15 \mathrm{~nm}$ by covering them with a microscope glass cover slip (cleaned with piranha solution, rinsed with DI water, and dried with nitrogen immediately before use) on which a weight of $1.7 \mathrm{~kg}$ was placed, and the whole setup was heated to $120^{\circ} \mathrm{C}$ under vacuum overnight.

Nanoindentation was performed using a scanning nanoindenter (Hysitron Triboindenter, Minneapolis, MN) system. Indentations were performed with a Berkovich diamond indenter with a tip radius of approximately $120 \mathrm{~nm}$. Prior to performing the indentations, the tip was calibrated using a fused silica reference sample. ${ }^{12}$ Load controlled indentations were performed with 5 second loading/unloading segments and a 10 second hold time at a peak load of $300 \mu \mathrm{N}$. Resulting indentation depths varied between 200-2700 nm. Samples were several hundred 
micrometers thick and several millimeters in the surface plane, so indentations sampled $<5 \%$ of the sample thickness. Furthermore, indentations to $1000 \mu \mathrm{N}$ returned the same values, indicating that the surrounding epoxy matrix did not significantly influence the results. At the same time, the minimum indentation diameter was about $1.5 \mu \mathrm{m}$, much larger than the nanostructure feature size so that the hardness and modulus values represent the composite behavior of the nanostructure.

The reduced elastic modulus $\mathrm{E}_{\mathrm{r}}$ and the hardness of the samples were determined through analysis of the unloading segments of the load displacement plots. ${ }^{12}$ The materials displayed a time-dependent behavior resulting in continued deformation in the forward direction during the initial part of unloading. In order to account for this behavior, the initial non-elastic part of the unloading curve was neglected for the determination of the mechanical properties. We found that a power law relation could be fit to the data between 20 and $75 \%$ of the maximum load (60-225 $\mu \mathrm{N})$ with very small errors $\left(\mathrm{R}^{2} \geq 0.98\right)$. We therefore assumed that this part of the curve represents elastic behavior and used the tangent to the power law fit at the maximum load as the contact stiffness to find the hardness and reduced modulus for each indentation. Although we cannot guarantee that this analysis fully eliminates viscoelastic effects, the calculated moduli of the samples should be quite comparable to each other since all analyses were performed identically.

The indentation modulus $\left(\mathrm{E}_{\text {indent }}\right)$ was then calculated from the reduced modulus $\left(\mathrm{E}_{\mathrm{r}}\right)$ according to the equation:

$$
E_{\text {indent }}=\left(\frac{E_{\text {sample }}}{1-v_{\text {sample }}^{2}}\right)=\left(\frac{1}{E_{r}}-\frac{1-v_{\text {tip }}^{2}}{E_{\text {tip }}}\right)^{-1}
$$

where $v_{\text {sample }}$ and $v_{\text {tip }}$ are the Poisson's ratios of the sample and the tip, respectively, and $E_{\text {sample, }}$ $\mathrm{E}_{\mathrm{tip}}$, and $\mathrm{E}_{\mathrm{r}}$ are the elastic moduli of the sample, the tip, and the reduced elastic modulus of the sample, respectively. The values $\mathrm{E}_{\text {tip }}=1220 \mathrm{GPa}, v_{\text {tip }}=0.2$ were used for the calculations. 


\section{d. Calculation of the volume fraction of nanoparticles in the hybrids}

Guessing a density of $\rho_{\mathrm{NP}}=2 \mathrm{~g} \mathrm{~cm}^{-3}$ for the nanoparticles, and using $\rho_{\text {PDMAEMA }}=1.15 \mathrm{~g} \mathrm{~cm}^{-3}$ for PDMAEMA, ${ }^{13,14}$ and $\rho_{\mathrm{PI}}=0.91 \mathrm{~g} \mathrm{~cm}^{-3}$ for $\mathrm{PI}^{15}$, the volume fractions of the nanoparticles were calculated according to

$$
\text { volume fraction of } N P=\frac{\frac{w t \%(N P)}{\rho_{N P}}}{\frac{w t \%(N P)}{\rho_{N P}}+\frac{w t \%(P D M A E M A)}{\rho_{P D M A E M A}}+\frac{w t \%(P I)}{\rho_{P I}}}
$$

where wt $\%(\mathrm{NP})$, wt $\%$ (PDMAEMA), and wt $\%(\mathrm{PI})$ were the weight fractions of the nanoparticles, PDMAEMA, and PI, respectively, and $\rho_{\mathrm{NP}}, \rho_{\mathrm{PDMAEMA}}, \rho_{\mathrm{PI}}$ were the densities of the nanoparticles, PDMAEMA, and PI, respectively. 


\section{References}

(1) Fayon, F.; Duee, C.; Poumeyrol, T.; Allix, M.; Massiot, D.: Evidence of Nanometric-Sized Phosphate Clusters in Bioactive Glasses As Revealed by Solid-State 31P NMR. J. Phys. Chem. C 2013, 117, 2283-2288.

(2) Leonova, E.; Izquierdo-Barba, I.; Arcos, D.; Lopez-Noriega, A.; Hedin, N.; Vallet-Regi, M.; Eden, M.: Multinuclear Solid-State NMR Studies of Ordered Mesoporous Bioactive Glasses. J. Phys. Chem. C 2008, 112, 5552-5562.

(3) Mathew, R.; Turdean-Ionescu, C.; Stevensson, B.; Izquierdo-Barba, I.; Garcia, A.; Arcos, D.; Vallet-Regi, M.; Eden, M.: Direct Probing of the Phosphate-Ion Distribution in Bioactive Silicate Glasses by Solid-State NMR: Evidence for Transitions between Random/Clustered Scenarios. Chem. Mater. 2013, 25, 1877-1885.

(4) Burkett, S. L.; Sims, S. D.; Mann, S.: Synthesis of hybrid inorganic-organic mesoporous silica by co-condensation of siloxane and organosiloxane precursors. Chem. Commun. 1996, 1367-1368.

(5) Zhang, P.; Grandinetti, P. J.; Stebbins, J. F.: Anionic Species Determination in CaSiO3 Glass Using Two-Dimensional 29Si NMR. J. Phys. Chem. B 1997, 101, 4004-4008.

(6) Ivankovic, M.; Brnardic, I.; Ivankovic, H.; Huskic, M.; Gajovic, A.: Preparation and properties of organic-inorganic hybrids based on poly(methyl methacrylate) and sol-gel polymerized 3-glycidyloxypropyltrimethoxysilane. Polymer 2009, 50, 2544-2550.

(7) Poologasundarampillai, G.; Ionescu, C.; Tsigkou, O.; Murugesan, M.; Hill, R. G.; Stevens, M. M.; Hanna, J. V.; Smith, M. E.; Jones, J. R.: Synthesis of bioactive class II poly(gamma-glutamic acid)/silica hybrids for bone regeneration. J. Mater. Chem. 2010, 20, 8952-8961.

(8) Schacher, F.; Muellner, M.; Schmalz, H.; Mueller, A. H. E.: New block copolymers with poly(N,N-dimethylaminoethyl methacrylate) as a double stimuli-responsive block. Macromol. Chem. Phys. 2009, 210, 256-262.

(9) Finnefrock, A. C.; Ulrich, R.; Toombes, G. E. S.; Gruner, S. M.; Wiesner, U.: The plumber's nightmare: A new morphology in block copolymer-ceramic nanocomposites and mesoporous aluminosilicates. J. Am. Chem. Soc. 2003, 125, 13084-13093.

(10) Toombes, G. E. S.; Finnefrock, A. C.; Tate, M. W.; Ulrich, R.; Wiesner, U.; Gruner, S. M.: A re-evaluation of the morphology of a bicontinuous block copolymer-ceramic material. Macromolecules 2007, 40, 8974-8982.

(11) Toombes, G. E. S.: Structural studies of block copolymer and block copolymer/aluminosilicate materials. Ph.D. Thesis, Cornell University, 2007.

(12) Oliver, W. C.; Pharr, G. M.: Measurement of hardness and elastic modulus by instrumented indentation: advances in understanding and refinements to methodology. J. Mater. Res. 2004, 19, 3-20.

(13) Stefik, M.; Wang, S.; Hovden, R.; Sai, H.; Tate, M. W.; Muller, D. A.; Steiner, U.; Gruner, S. M.; Wiesner, U.: Networked and chiral nanocomposites from ABC triblock 
terpolymer coassembly with transition metal oxide nanoparticles. J. Mater. Chem. 2012, 22, 1078-1087.

(14) Slim, C.; Tran, Y.; Chehimi, M. M.; Garraud, N.; Roger, J.-P.; Combellas, C.; Kanoufi, F.: Microelectrochemical Patterning of Surfaces with Polymer Brushes. Chem. Mater. 2008, 20, 6677-6685.

(15) Brandrup, J.; Immergut, E. H.; Grulke, E. A.: Polymer handbook; 4th ed.; Wiley: New York, 1999. 